\title{
Pattern-induced magnetic anisotropy in FePt thin films by ion irradiation
}

\author{
M. Jaafar, ${ }^{1,2, *}$ R. Sanz, ${ }^{1,3}$ J. McCord, ${ }^{4}$ J. Jensen, ${ }^{5}$ R. Schäfer, ${ }^{6}$ M. Vázquez, ${ }^{1}$ and A. Asenjo ${ }^{1}$ \\ ${ }^{1}$ Instituto de Ciencia de Materiales de Madrid, CSIC, ES-28049 Madrid, Spain \\ ${ }^{2}$ Dpto. Física de la Materia Condensada, UAM, ES-28049 Madrid, Spain \\ ${ }^{3}$ Nanoate SL, Poeta Rafael Morales 2, ES-28702 San Sebastián de los Reyes, Madrid, Spain \\ ${ }^{4}$ Institute of Ion Beam Physics and Materials Research, FZD, D-01314 Dresden, Germany \\ ${ }^{5}$ Thin Film Physics, Department of Physics, Chemistry and Biology, Linköping University, SE-581 83 Linköping, Sweden \\ ${ }^{6}$ Leibniz Institute for Solid State and Materials Research, IFW, Helmholtzstrasse 20, D-01069 Dresden, Germany
}

(Received 28 June 2010; published 23 March 2011)

\begin{abstract}
The magnetic properties of FePt thin films have been modified by exposing the samples to irradiation of $4 \mathrm{MeV} \mathrm{Cl}^{2+}$ ions. Patterned magnetic films, without modified topographical profile, were fabricated by irradiating the films through a shadowing micrometric mask. The structural changes, ascribed to the ion-beam-induced amorphization of the thin films, promote the modification of the magnetic anisotropy. In particular, the out-of-plane component of the magnetization decreases simultaneously with an enhancement of in-plane anisotropy by increasing ion fluence. Moreover, the nonirradiated regions present unexpected anisotropic behavior owing to the stray field of the irradiated regions. The control of this effect, which can have unwished consequences for the patterning of magnetic properties by ion bombardment, needs to be suitably addressed.
\end{abstract}

DOI: 10.1103/PhysRevB.83.094422

PACS number(s): 68.37.Rt, 75.50.-y, 61.80.-x

\section{INTRODUCTION}

In the last few years, great efforts have been made in the miniaturization of magnetic systems. The understanding of the magnetic properties of such micro- or nanosystems is crucial because they display novel magnetic behaviors, which are associated with low-dimensional effects. These systems can be used in a variety of applications such as magnetic storage, semiconductor industries, or ultra-small magnetic field sensors. For magnetic thin films, several methods are currently employed to produce patterned media: self-organization and self-assembly of nanoelements, ${ }^{1}$ electron beam lithography, ${ }^{2}$ focused electron-beam-induced deposition, ${ }^{3}$ or ion beam irradiation. ${ }^{4,5}$

The effects of local ion irradiation have been studied previously, and for a review, the reader is addressed to Refs. 6 and 7. In particular, an initial study on the effect of ion irradiation in FePt thin films was previously reported by the authors. ${ }^{8}$ The aim of this work is to study the effects of highenergy ion beam irradiation on the structural and magnetic properties of FePt thin films. ${ }^{4,9,10}$ Since the irradiation was performed through a micrometric mask, a magnetic film patterned at the micrometer scale was obtained., ${ }^{4,11,12} \mathrm{We}$ report a study on the induced magnetic anisotropy in $\mathrm{FePt}$ samples after bombardment with $4 \mathrm{MeV} \mathrm{Cl}^{2+}$ ions varying the ion fluence between $1.0 \times 10^{13}$ and $4.0 \times 10^{14}$ ions $\mathrm{cm}^{-2}$. From the magnetic characterization we conclude that in-plane anisotropy develops during the ion beam patterning process.

\section{EXPERIMENT}

Polycrystalline FePt thin films $35 \mathrm{~nm}$ thick have been grown by Radio Frequency (RF) magnetron sputtering onto $\mathrm{Si}(100)$ at $600^{\circ} \mathrm{C}$ with a Pt buffer layer (28 nm thick). The structural and magnetic properties of the films were determined by grazing incidence X-ray diffraction (GI-XRD), Rutherford backscattering spectrometry (RBS) in random mode, atomic force microscopy (AFM), vibrating sample magnetometer (VSM), Kerr magnetometry, and magnetic force microscopy (MFM) under an in-plane externally applied magnetic field. ${ }^{13,14}$

For the as-prepared film, transmission electron microscopy (TEM) and XRD analysis were performed in a previous work. ${ }^{8}$ The TEM micrographs show a columnar growth of the layer with crystallite sizes of about $20 \mathrm{~nm}$. Also, as can be inferred from the XRD data, the polycrystalline FePt thin film grows preferentially in an FCC structure with certain (111) fiber texture and no in-plane structural order. As a consequence, the sample does not present high uniaxial anisotropy, and mainly in-plane anisotropy is expected due to the shape anisotropy. In addition, as concluded from the magnetization curves obtained by VSM, the films mainly exhibit in-plane anisotropy with a small out-of-plane anisotropy contribution $(m r \perp \approx 4.5 \%)$ in good agreement with the structural information (see Ref. 8 for more information).

An AFM Cervantes system (Nanotec Electronica S.L.) was used to characterize the topography (AFM mode) and the local magnetic properties (MFM mode) of the sample. The images were processed with WSxM software. ${ }^{15}$ Co-coated Veeco Mesp LM cantilevers were used. The stiffness and resonance frequency of the cantilevers used for the experiments (always in dynamic mode) were $k=1.5 \mathrm{~N} / \mathrm{m}, f_{0}=69 \mathrm{kHz}$. From the MFM images, a dense stripe domain configuration-related to the weak out-of-plane anisotropy-was deduced. It should be noticed that in the as-grown sample no in-plane easy axis was detected. Moreover, MFM images under external magnetic fields varying between \pm 600 Oe were obtained by using variable-field MFM (VFMFM). ${ }^{12}$ Kerr effect magnetometry was performed in longitudinal mode.

A set of irradiated FePt samples was prepared by varying the $\mathrm{Cl}^{2+}$ ions' fluence, from $1.0 \times 10^{13}$ to $4.0 \times 10^{14}$ ions $/ \mathrm{cm}^{2}$ as shown in Table I. Sample I denotes the as-grown thin film used as a reference. The magnetic thin films were exposed to $4 \mathrm{MeV} \mathrm{Cl}^{2+}$ ions delivered from the tandem accelerator at Uppsala University. The irradiation was performed through a $\mathrm{Cu}$ mask $0.8 \mathrm{~mm}$ in thickness, with an array of square holes 
TABLE I. Irradiation parameters (ion fluence, size of the square hole of the mask, $\%$ of the sample that have been irradiated) and in-plane coercivity of irradiated and nonirradiated areas measured by VSM.

\begin{tabular}{llclr}
\hline \hline Sample & $\begin{array}{l}\text { Fluence } \\
\left(\text { ions } / \mathrm{cm}^{2}\right)\end{array}$ & $\begin{array}{c}\text { Hole side } \\
(\mu \mathrm{m})\end{array}$ & $\begin{array}{l}\text { Irradiated } \\
\text { sample }(\%)\end{array}$ & $\begin{array}{r}H c_{\|} \\
(\mathrm{Oe})\end{array}$ \\
\hline I & as-grown & - & - & 370 \\
II & $0.1 \times 10^{14}$ & 7 & 70 & 340 \\
III & $0.7 \times 10^{14}$ & 113 & 80 & 175 \\
IV & $2.2 \times 10^{14}$ & 95 & 60 & 30 \\
V & $4.0 \times 10^{14}$ & 45 & 85 & 25 \\
\hline \hline
\end{tabular}

tens of microns on a side (see Table I), in order to fabricate a micropatterned samples. The irradiations were performed at room temperature under normal incidence with respect to the samples' surface. A homogenous irradiation over the samples was achieved by means of an electrostatic raster scanner. The ion fluence was determined from the irradiation time and the beam current, which was measured using a Faraday cup located behind the sample holder. The beam flux was low enough to prevent direct heating of the samples during ion bombardment.

The effects of the sample irradiation were simulated by using the SRIM2006 ${ }^{16}$ code. Those Monte Carlo simulations indicate that the irradiation does not reach the areas of the thin film that are covered by the solid part of the $\mathrm{Cu}$ mask. Therefore, only a certain percentage of the total surface (corresponding to the mask holes) is exposed to the irradiation, as shown in Table I. Although the size of the induced pattern could be expected to result in a modification of the magnetic properties, ${ }^{17}$ no relationship between the properties of the irradiated regions and the size of the mask holes was found in the range of the experiment. However, a clear change of behavior is observed when submicron patterning is performed under the same conditions. ${ }^{18}$

According to the SRIM simulations, the ions have a range of $\sim 1 \mu \mathrm{m}$, which is larger than the FePt film thickness. Therefore, in the areas directly exposed to ion bombardment, no implantation of ions in the magnetic material is expected. Also the nature of the employed ions does not induce additional magnetic effects into the Si substrate. The slowing down of the ions in the film is mainly governed by the electronic stopping power, although the nuclear stopping is not insignificant. However, sputtering effects at the surface are considered negligible.

\section{RESULTS AND DISCUSSION}

\section{A. Macroscopic characterization}

From the structural characterization by XRD significant damage in the irradiated films can be inferred. The irradiation promotes structural damage of the sample, ${ }^{7,8}$ i.e., the $\mathrm{Si}$ substrate, the Pt buffer layer, and the FePt thin film, as deduced from the $\theta-2 \theta$ XRD scan and grazing XRD data. As observed in the inset of Fig. 1, the GI-XRD diffraction peaks decreases, and some of them nearly disappear, with increasing ion fluence. This behavior is believed to be related with the accumulative build-up of damage or disorder with increasing fluence.

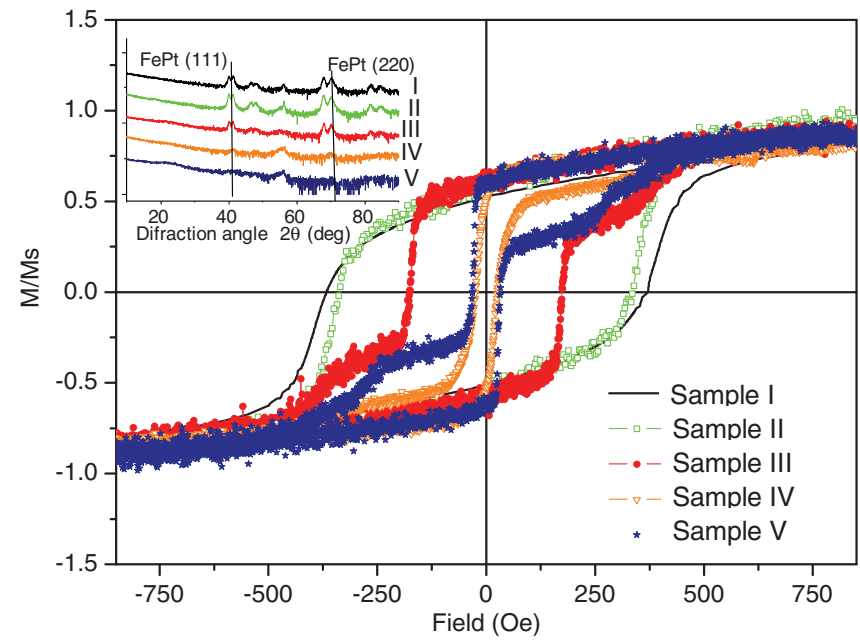

FIG. 1. (Color online) In-plane hysteresis loops of the samples performed by VSM as a function of the irradiation parameters. Inset shows grazing XRD data.

The damage build-up is often described with a well-known defect overlap model, ${ }^{19}$ which takes into account a spatial overlap of regions with an incomplete defect or damage structure created around each ion impact or track. One can thus assume that the high-energy ions create highly affected cylindrical volume damage in the form of displacements of atoms, interstitials, and vacancies through elastic atomic collisions. ${ }^{20}$ Accumulation of this kind of damage with increasing ion fluence would thus result in a highly defective film. Nevertheless, the situation becomes more complex by the fact that the $4 \mathrm{MeV} \mathrm{Cl}$ ions also accumulate a large amount of energy into excitation and ionization, which may contribute to or even dominate the creation of defects through latent track formation. ${ }^{21}$ We thus believe that the irradiated film becomes essentially amorphous (or highly defective with too small nanocrystals to be detected in XRD) for the highest ion fluence used. It is important to notice that the presence of some weak peaks in XRD data is a consequence of the amorphitation process. For example, at the (201) FePt position it seems to be a peak that is forbidden in an FCC structure This is not a real peak but an amorphous halo.

RBS measurements performed in random mode, using $2 \mathrm{MeV} \mathrm{He}^{+}$ions, of the irradiated samples (not shown here) do not show any clear change in film composition and thickness compared to the as-deposited sample.

As a consequence of the structural changes, a subsequent modification of the magnetic anisotropy as a function of the ion fluence is expected. The hysteresis loops for samples irradiated with different ion fluence, measured by VSM, are shown in Fig. 1. Their modification with increasing ion fluence confirms the significant effect of structural changes on the magnetic behavior.

The magnetization curve of irradiated samples presents a two-step magnetization reversal process typically corresponding to the irreversible process ascribed to the irradiated and nonirradiated regions, respectively. Irreversibility occurring at a higher critical field, near the coercivity of the asgrown sample $\left(H_{c}^{0}\right)$, corresponds to the contribution of the nonirradiated regions. In addition, an important conclusion 

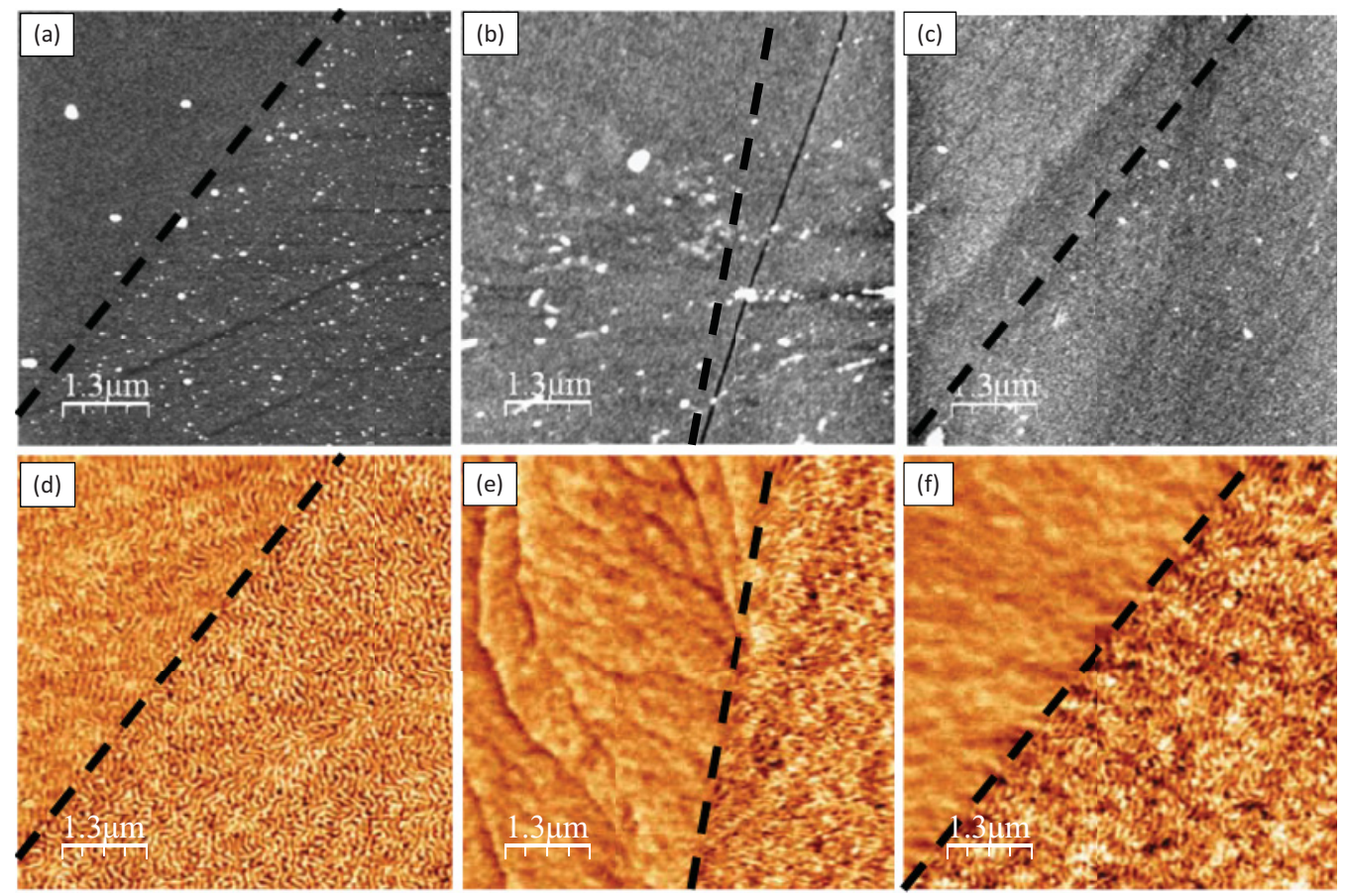

FIG. 2. (Color online) Topography $(\mathrm{a}-\mathrm{c})$ and MFM images $(\mathrm{d}-\mathrm{f})$ obtained in remanence state corresponding to the boundary zone between irradiated (left) and nonirradiated (right) regions in sample III (a and d), sample IV (b and e), and sample V (c and f). The dashed line is the boundary between irradiated and nonirradiated areas. The size of the images is $6.5 \mu \mathrm{m} \times 6.5 \mu \mathrm{m}$.

deduced from the VSM measurements is that the lower critical field corresponding to the irradiated areas depends on the ion fluence. In particular, when the ion fluence increases, a decrease of coercivity is observed (see data collected in Table I). Notice that the fractional volume of the contribution of both areas to the total VSM signal depends on the relative surface area exposed to the irradiation; see Table I.

\section{B. Scanning force microscopy characterization}

In order to study the interface region between the irradiated and nonirradiated regions, atomic and magnetic force microscopy images were obtained. MFM imaging, taken in remanence state [Fig. 2(d)-2(f)], confirms the existence of the two regions with different magnetic configuration corresponding to the irradiated (left) and nonirradiated (right) areas. Interpretation of these magnetic images agrees with the different magnetic character deduced from the hysteresis loops. However, no differences between these areas were observed in the corresponding topographic images [Fig. 2(a)(c)] Moreover, no significant change of the roughness of the thin film surface due to the irradiation has been observed. This so-called "magnetic patterning," where only the magnetic properties are tailored ${ }^{22}$ is more suitable for some applications than patterning where both topography and magnetic behavior are modified. ${ }^{23}$

The presence of an out-of-plane component of the anisotropy in the as-grown sample, and hence in the nonirradiated areas, reveals on the development of the so-called dense stripe domains ${ }^{24}$ observed in the right part of MFM images in Figs. 2(d)-(f). Mainly, this out-of-plane component of the magnetization is modified by the ion irradiation as deduced from the hysteresis loops shown above. The MFM image in Fig. 2(d), corresponding to a low fluence irradiation (sample III), shows a decrease of the dense stripe domain contrast in the irradiated region. Moreover, this kind of contrast even disappears for higher irradiation fluence, as shown in Fig. 2(e) and Fig. 2(f) (corresponding to samples IV and V, respectively). In these latter samples, instead of dense stripes, the irradiated regions present in-plane domains as observed in the MFM images. A progressive reduction of the out-of-plane component is thus concluded when increasing the ion fluence.

As shown in Fig. 1, the magnetization process presents some differences as a function of the ion fluence. To further understanding of the magnetization process in the irradiated and nonirradiated regions, magnetic images were taken under variable applied magnetic field (VFMFM) imaging. Figure 3 corresponds to a series of MFM images of the boundary between irradiated and nonirradiated regions in samples III and $\mathrm{V}$ obtained under different in-plane external magnetic fields applied in situ. In the case of sample III, MFM imaging study shows the evolution of the dense stripe domains in both regions. Although the reversal magnetization process in both regions is similar, the critical fields in the irradiated regions differ, however, from the observed in the as-grown areas. Dense stripe domains disappear at lower fields in the regions exposed to the ion damage due to the reduction of the strength of the out-of-plane anisotropy component [Fig. 3(a)-3(d)]. Notice that the dense stripe domains of the irradiated area disappear at magnetic fields about 400 Oe. However, the dense stripe domains in the nonirradiated regions remain up to fields near the $H_{c}^{0}$. No pinning effects were observed in the boundary region.

The dense stripe domains do not appear at all in the irradiated regions with higher ion fluence, as shown in Fig. 2. 

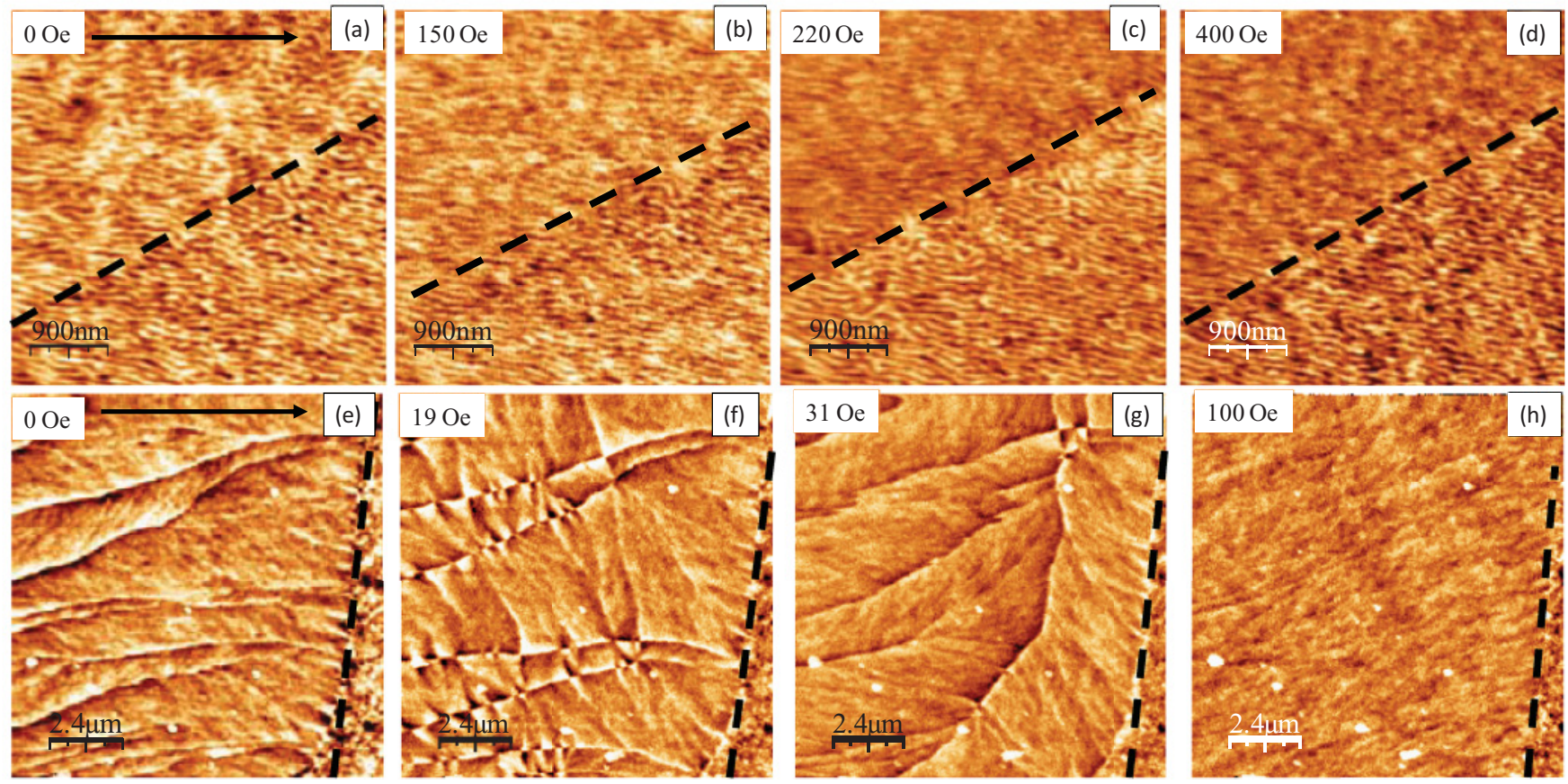

FIG. 3. (Color online) (a-d) MFM images of sample III obtained in the boundary (marked with a dashed line) between the irradiated (top left) and nonirradiated (lower right) regions under different values of in plane magnetic fields: (a) 0 Oe, (b) 150 Oe, (c) 220 Oe, and (d) 400 Oe. Image size: $4.5 \mu \mathrm{m} \times 4.5 \mu \mathrm{m}$. (e-h) MFM images of sample V obtained in the boundary between the irradiated (left) and nonirradiated (right) regions under different values of in-plane magnetic fields: (e) 0 Oe, (f) 19 Oe, (g) $31 \mathrm{Oe}$, and (h) 100 Oe. Image size: $12 \mu \mathrm{m} \times 12 \mu \mathrm{m}$.

The MFM images of sample V, shown in Fig. 3(e)-3(h), confirm the disappearance of the dense stripe domains due to the decrease of the out-of-plane anisotropy-and thus the outof-plane component of the magnetization-in the irradiated region. The reversal magnetization mechanism in this sample is based on the nucleation and propagation of domain wall inside the modified region. When the field increases the propagation of cross-tie domain walls can be followed [see Fig. 3(e)-3(h)]. In agreement with the VSM hysteresis loops, the irradiated region is nearly saturated under a magnetic field lower than $100 \mathrm{Oe}$, much lower than the field, $H_{c}^{0}$, required to start approaching to saturation of the nonirradiated areas. In this case, wall-pinning effects are observed in the boundary region.

\section{Kerr magnetometry characterization}

The VSM data as well as the MFM images recorded under an external magnetic field inform us about the existence of two regions with different magnetic properties, i.e., two critical fields for magnetization reversal and different out-of-plane component. For a full quantitatively determination of the local coercive field in the irradiated and nonirradiated areas, separate Kerr magnetometry analysis was performed. The hysteresis loops were obtained electronically from the longitudinal Kerr microscope images recorded at different fields. The image intensity in a selected area (always with the same size) was integrated and plotted as a function of the magnetic field. The Kerr effect hysteresis loops in Fig. 4(a) and 4(b) corresponding to three different samples (III, IV, and V) confirm a noticeable reduction of the coercive field in the irradiated, together with a moderate decrease of the coercive field in nonirradiated, area, as increasing the fluence. In particular, a nearly exponential decay of the coercive field in the irradiated areas as a function of the total ion fluence has been found; ${ }^{25}$ see Fig. 4(c). Fitting the curve to equation

$$
H_{c}=H_{c}^{0} e^{-\beta \Phi},
$$

where $H_{c}^{0}$ is the coercivity of the unexposed film, $\Phi$ is the ion fluence (given in ions $/ \mathrm{cm}^{2}$ ), and $\beta$ is the fitted constant, we obtain a value of $\beta=1.25 \times 10^{-14} \mathrm{~cm}^{2} /$ ion. This value is quite similar to the data obtained by Rettner et al., ${ }^{22}$ where the decrease in coercivities with ion irradiation fluence in high-anisotropy magnetic multilayers was interpreted to be a consequence of interface intermixing induced by elastic collisions.

In the present case, the observed decrease in the coercivity with ion fluence could be related with the accumulated disorder created around each ion track. Assuming the tracks occupy part of the sample volume according to the Poisson law, the normalized decrease in coercivity may thus be expressed as Eq. (1), where $\beta$ would denote the area of the defective region projected on the surface of the sample. From the obtained fitted $\beta$ value one can extract a mean damage diameter of $\sim 6 \AA$. The nature of the damage formation leading to the observed disordering of the thin films and thereby to a decrease the in-plane coercivity is currently not fully understood. The origin of the structural and thus magnetic changes observed in the irradiated regions can be due to a combination of damage effects such as elastic collisions, ionization, and electronic excitations. SRIM simulations indicate that the main processes promoted by the irradiation are the inelastic collisions rather than direct elastic displacements. Whatever 

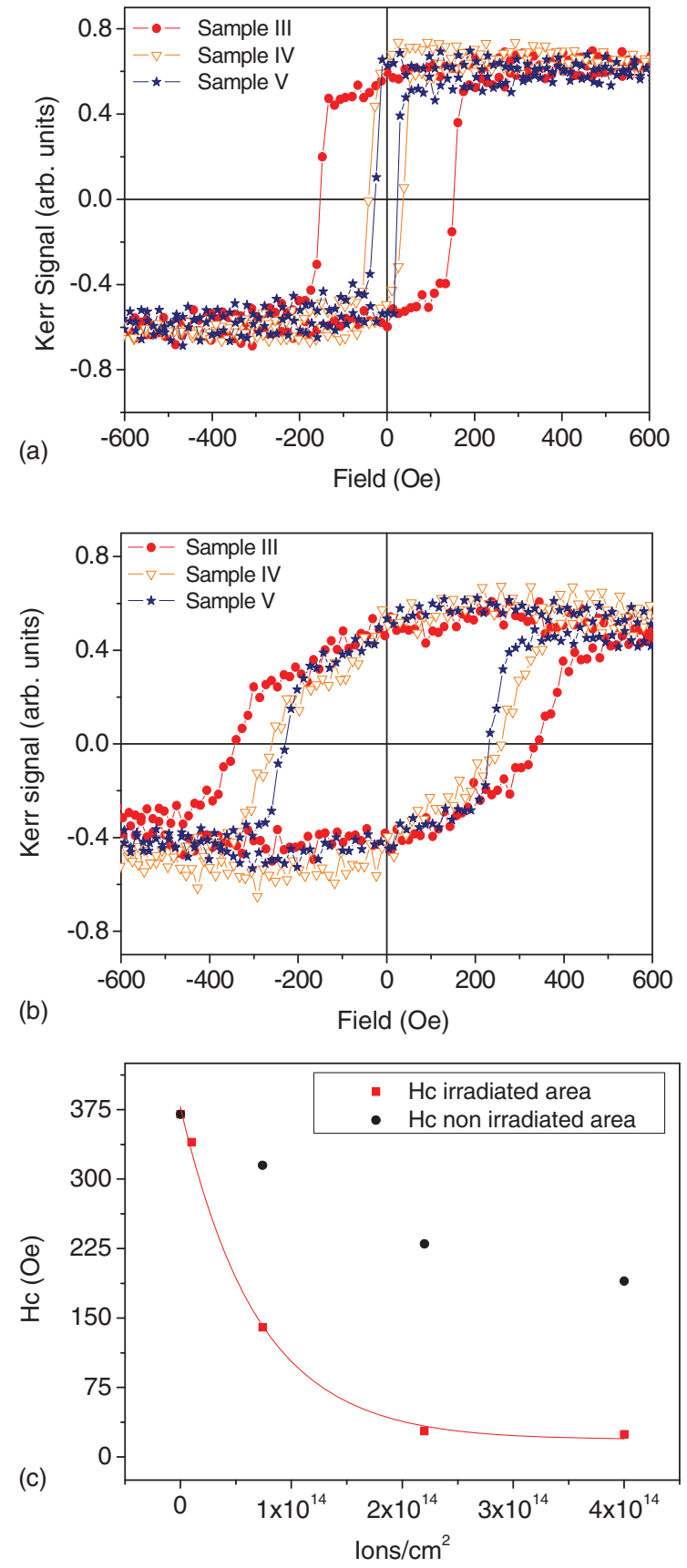

FIG. 4. (Color online) (a) Kerr magnetometry hysteresis loops corresponding to the irradiated and (b) nonirradiated regions. (c) Coercive fields as a function of the total fluence. The data corresponding to the irradiated region are fitted to an exponential decay.

process is responsible for the observed damage formation, a progressive amorphization of the thin film is supported by the XRD data and magnetic characterization.

A noticeable and intriguing observation is the unexpected evolution of coercivity in the nonirradiated regions [see Fig. 4(c)]. The coercivity decays with the ion fluence in a similar trend as for the irradiated regions, indicating seemingly some connection between both decay mechanisms. This decay is nevertheless not as fast as in the irradiated areas. This behavior could be related with the development of nucleation centers at the boundaries of the irradiated areas and with

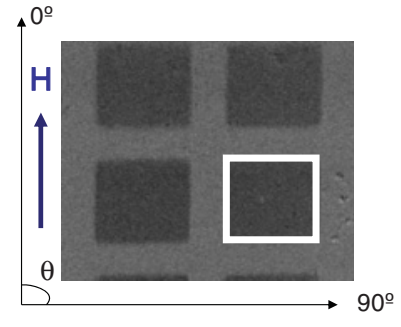

(a)

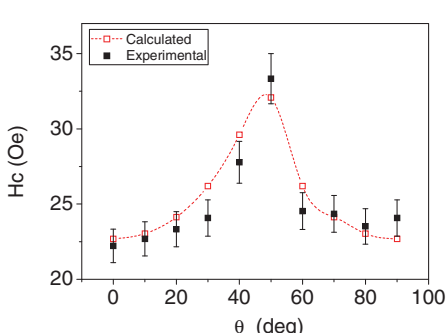

FIG. 5. (Color online) Scheme of the geometry of the magnetic field applied. (a) Kerr image of sample V obtained under 130 Oe applied magnetic field $(\Theta=0)$. Image size $170 \mu \mathrm{m} \times 130 \mu \mathrm{m}$. (b) Experimental and calculated angular dependence of coercivity of the irradiated regions as a function of the applied field direction.

the magnetostatic interactions between neighboring irradiated regions. Further analyze of this effect will be considered in the next section.

\section{Induced anisotropy}

A systematic study about the local reversal magnetization process in both irradiated and nonirradiated regions was performed with a Kerr microscope. To evaluate the angular dependence of the reversal magnetization process in the irradiated regions, the sample was rotated, keeping constant the magnetic field applied along the sensitivity direction of the Kerr microscope. Then the magnetic field (with amplitude varying between \pm 600 Oe) was applied along different orientations of the square side (see Fig. 5).

The analysis of the reversal magnetization process from the Kerr images, and particularly that of coercivity, indicates the presence of a magnetic anisotropy associated with shape effects. Coercivity, $H_{c}$, is phenomenologically observed to depend inversely on the cosine of the angle $\theta$ between the applied magnetic field and the lateral side of the square patterned area as shown in Fig. 5(b). ${ }^{26}$ This angular dependence of coercivity can be justified by the presence of an effective biaxial magnetic anisotropy at $90^{\circ}$ ascribed to the easy axes determined by the square shape of the irradiated regions. The prevalence of shape anisotropy is understood to be a consequence of the amorphous character of such irradiated regions. Good agreement between the experimental and calculated values is obtained.

Concerning the nonirradiated areas, three different regions can be distinguished in the Kerr images obtained under different magnetic fields applied along the $\Theta=0$ direction. Those regions marked in Fig. 6(a) as A, B, and $\mathrm{C}$ present distinct magnetic behavior. In particular, as observed in Fig. 6(b), in the $B$ and $C$ regions magnetization reverses at critical fields higher than in region A. Similar behavior has been observed in all the samples, and in particular, this effect is enhanced with increasing the ion fluence as observed in Fig. 6(c). Hence, we conclude that ion irradiation induces local shape magnetic anisotropy, as deduced also from the observed anisotropy in the nonirradiated zones. ${ }^{27}$

Applying the magnetic field in a different $\Theta$ direction with respect to the square side [see Fig. 6(d)] and following the reversal magnetization process from the Kerr images, we evaluate the coercivity in the nonirradiated A zone. We observe 


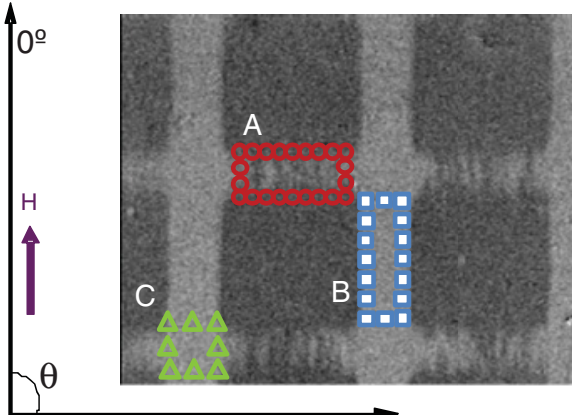

(a)

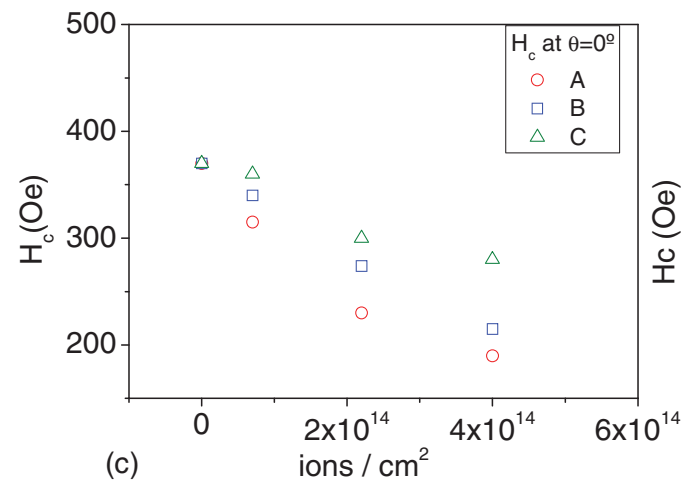

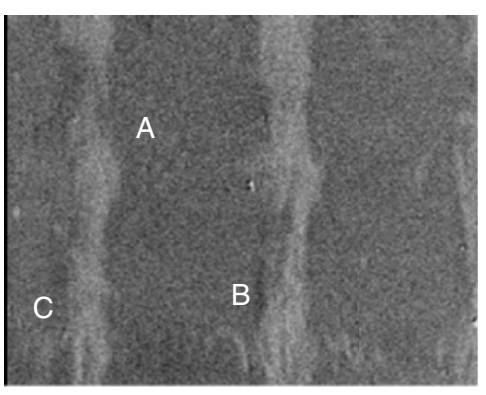

(b)

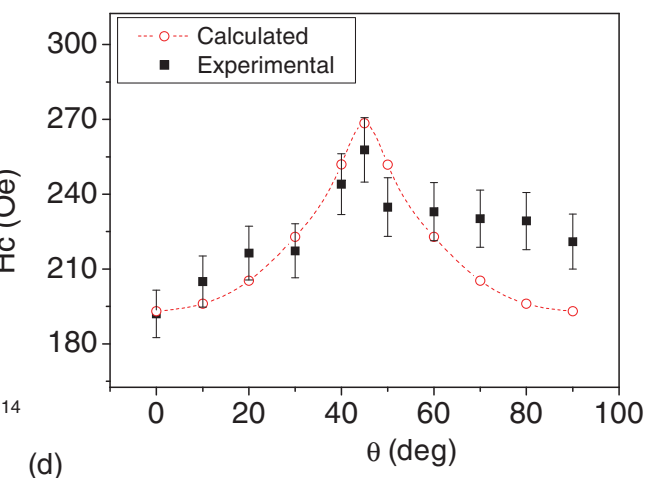

FIG. 6. (Color online) Scheme of the geometry of the magnetic field applied. Kerr images of sample V obtained under an external magnetic field of (a) $210 \mathrm{Oe}(\Theta=0)$ and (b) $220 \mathrm{Oe}(\Theta=0)$. Image size $170 \mu \mathrm{m} \times 130 \mu \mathrm{m}$. (c) Hc measured in the different nonirradiated regions A, B, and C [marked on (a)] of samples I, III, IV, and V, respectively. (d) Experimental and calculated values of the coercitive fields of sample $\mathrm{V}$ as a function of the applied field direction in nonirradiated A region.

an increase of the coercive field when the $\Theta$ angle increases up to $45^{\circ}$, similar to the behavior observed in the irradiated regions [Fig. 5(b)]. However, for higher angles, an asymmetric response is observed; i.e., the coercive field in the nonirradiated A area does not fit to the calculated curve as occurs in the previous case. The origin of such behavior is ascribed to the magnetostatic interactions between neighboring regions and the geometry of the magnetic patterning. Moreover it is important to mention the importance of exchange coupling in these patterned materials. ${ }^{28}$ At the border between irradiated and nonirradiated samples, as an effect of the proximity of the two phases, we might expect an exchange coupling. Such a coupling should be more important in the case of the samples with higher fluence due to drastic reduction of the coercive field. Nevertheless, since the material is separated in domains, the exchange coupling effect can be limited to the borders and then masked by the magnetostatic interaction. This conclusion is supported by the angular dependence of the coercive field (see Fig. 6). Here again the exchange coupling can also be taken into account since it also depends on the lateral size of the patterns.

It is thus crucial to consider this unexpected change in the magnetic properties of the nonirradiated regions to obtain a patterned magnetic film by high-energy ions' irradiation, using, e.g., lithographic methods. ${ }^{29}$ This local shape anisotropy is governed by the relative position of the irradiated zones through magnetostatic interactions. Due to such magnetostatic interaction there is a limitation in the separation between the irradiated regions to avoid a collapse between both regions, which is a function of the ion fluence. Finally, it should be noted that the size of nonirradiated areas in the present case is in the micrometric range. Going down in the nanometer range would probably increase the problem.

\section{CONCLUSIONS}

In conclusion, irradiation of FePt thin films with $4 \mathrm{MeV}$ $\mathrm{Cl}^{2+}$ ions induces a magnetic softening of the irradiated region, which manifests in a reduction of the in-plane coercive field and the out-of-plane component of the anisotropy as deduced from the VSM and MFM data. This softening of the magnetic thin film is interpreted to be a consequence of the amorphization effect produced by the irradiation. As a consequence, such amorphization results in an enhancement of the relative contribution of shape against the crystalline anisotropy; hence the effective easy axis tends to move to the in-plane direction. Moreover, the coercivity of the samples decreases exponentially with the irradiation fluence. Due to the shape anisotropy, the coercive field in the irradiated regions is inversely proportional to the cosine of the angle between the magnetic field and the square sides.

An unexpected parallel change in the reversal magnetization process of the nonirradiated regions was also observed. The corresponding reduction of the coercivity is related with the magnetostatic interaction between the neighboring irradiated regions. In addition, this interaction induces the local in-plane shape anisotropy in the nonirradiated regions, which should be taken into account when using ion-beam-based 
patterning. However, due the high dependence with the magnetic field orientation, this kind of patterned material is suggested to be useful as a sensing element in sensor devices.

\section{ACKNOWLEDGMENTS}

The authors thank M. Hernández-Velez for his useful discussions. We gratefully acknowledge financial support from the Spanish Ministerio Ciencia e Innovación through the project MAT2007-65420-C02-01 and Comunidad Autónoma de Madrid grant S2009/MAT-1467. MJ thanks Comunidad Autónoma de Madrid and CSIC Marina Bueno Program for their financial support. RS acknowledges the Spanish Ministry of Education and Science for a FPI research grant. JJ acknowledges the financial support from Linköping University through the VR Linneaus grant LiLi-NFM *miriam.jaafar@uam.es

${ }^{1}$ T. Thomson, G. Hu, and B. D. Terris, Phys. Rev. Lett. 96, 257204 (2006).

${ }^{2}$ C. Vieu, F. Carcenac, A. Pépin, Y. Chen, M. Mejias, A. Lebib, L. Manin-Ferlazzo, L. Couraud, and H. Launois, Appl. Surf. Sci. 164, 111 (2000).

${ }^{3}$ W. F. Van Dorp, B. van Someren, C. K. Hagen, P. Kruit, and P. A. Crozier, Nano Lett. 5, 1303 (2005).

${ }^{4}$ C. Chappert et al., Science 280, 1919 (1998).

${ }_{5}^{5}$ S. Konings, J. Miguel, J. Goedkoop, J. Camarero, and J. Vogel, J. Appl. Phys. 100, 033904 (2006).

${ }^{6}$ J. Fassbender and J. McCord, J. Magn. Magn. Mater. 320, 579 (2008).

${ }^{7}$ J. Fassbender, D. Ravelosona, and Y. Samson, J. Phys. D 37, R179 (2004).

${ }^{8}$ M. Jaafar, R. Sanz, M. Vázquez, A. Asenjo, J. Jensen, K. Hjort, S. Flohrer, J. McCord, and R. Schäfer, Phys. Stat. Sol. A 204, 1724 (2007).

${ }^{9}$ F. Studer and M. Toulemonde, Nucl. Instrum. Methods B 65, 560 (1992).

${ }^{10}$ T. Hasegawa, W. Pei, T. Wang, Y. Fu, T. Washiya, H. Saito, and S. Ishio, Acta Mater. 56, 1564 (2008).

${ }^{11}$ B.D. Terris, L. Folks, D. Weller, J. E. E. Baglin, A. J. Kellock, H. Rothuizen, and P. Vettiger, Appl. Phys. Lett. 75, 403 (1999).

${ }^{12}$ J. McCord, T. Gemming, L. Schultz, J. Fassbender, M. O. Liedke, M. Frommberger, and E. Quandt, Appl. Phys. Lett. 86, 162502 (2005).

${ }^{13}$ A. Asenjo, D. García, J. M. García, C. Prados, and M. Vázquez, Phys. Rev. B 62, 6538 (2000).

${ }^{14}$ M. Jaafar, J. Gómez-Herrero, A. Gil, P. Ares, M. Vázquez, and A. Asenjo, Ultramicroscopy 109, 693 (2009).
${ }^{15}$ I. Horcas, R. Fernández, J. M. Gómez-Rodríguez, J. Colchero, J. Gómez-Herrero, and A. M. Baró, Rev. Sci. Instrum. 78, 013705 (2007).

${ }^{16}$ [http://www.srim.org].

${ }^{17}$ J. I. Martin, J. Magn. Magn. Mater. 256, 449 (2003).

${ }^{18}$ Samples irradiated through ordered alumina membrane with pores about $55 \mathrm{~nm}$ and a lattice parameter of $105 \mathrm{~nm}$ were also analyzed. In this case, and due to the importance of the pattern size the behavior is completely different and the $\mathrm{Hc}$ is a factor 2 smaller than the expected value (unpublished data).

${ }^{19}$ J. F. Gibbons, Proc. IEEE 60, 1062 (1972).

${ }^{20}$ Y. Zushi, M. Fukuzumi, Y. Chimi, N. Ishikawa, F. Ono, and A. Iwase, Nucl. Instrum. Methods B 256, 434 (2007).

${ }^{21}$ F. Ono, Y. Matsushima, Y. Chimi, N. Ishikawa, T. Kambara, and A. Iwase, J. Magn. Magn. Mater. 310, 1864 (2007).

${ }^{22}$ E. Menéndez et al., Small 5, 229 (2009).

${ }^{23}$ S. Y. Chou, M. S. Wei, P. R. Krauss, and P. Fischer, J. Appl. Phys. 76, 6673 (1994).

${ }^{24}$ A. Hubert and R. Schäfer, Magnetic Domains (Springer-Verlag, Berlin, 1998).

${ }^{25}$ C. T. Rettner, S. Anders, J. E. E. Baglin, T. Thomson, and B. D. Terris, Appl. Phys. Lett. 80, 279 (2002).

${ }^{26}$ We use the following function to calculate $H_{c}: H_{c}=H_{c}^{0} / \cos \Theta$ for $0 \leqslant \Theta \leqslant 45$ and $H_{c}=H_{c}^{0} / \sin \Theta$ for $45 \leqslant \Theta \leqslant 90$, where $H_{c}^{0}$ is the experimental value for $\Theta=0$.

${ }^{27}$ J. McCord, I. Mönch, J. Fassbender, A. Gerber, and E. Quandt, J. Phys. D: Appl. Phys. 42, 055006 (2009).

${ }^{28}$ J. McCord, L. Schultz, and J. Fassbender, Adv. Mater. 20, 2090 (2008).

${ }^{29}$ R. Sanz, A. Johansson, M. Skupinski, J. Jensen, G. Possnert, M. Boman, M. Vazquez, and K. Hjort, Nano Lett. 6, 1065 (2006). 